Ann. Zootech., I973, $22(2), 249-25$ I.

NOTE

\title{
POSSIBILITÉS D'ALIMENTATION DU LAPIN EN CROISSANCE AVEC DES RÉGIMES PRÉSENTÉS SOUS FORME DE FARINE
}

\author{
F. LEBAS \\ avec la collaboration technique de G. SARDI \\ Station de Recherches sur l'Élevage des Porcs, \\ Centre national de Recherches zootechniques, I. N.R. A., \\ 78350 Jouy en Josas

\section{RÉSUMÉ}

Deux régimes composés l'un de tourteau de soja, d'amidon de maïs, d'huile et de paille d'orge, l'autre de tourteau de soja, de maïs et de paille, ont été distribués, soit sous forme d'aggloméré, soit sous forme de farine, à un total de 32 lapereaux de 7 semaines. Les 2 aliments ont été bien consommés durant les 4 semaines d'expérience, quel que soit le type de présentation. Il semble que la granulation ait un effet favorable sur les performances de croissance. La réussite de l'alimentation des lapins avec un aliment en farine pourrait être due à l'absence de luzerne et de foin de graminées dans les régimes.

\section{INTRODUCTION}

Le lapin est actuellement alimenté, soit avec des fourrages ou céréales en nature, soit avec un aliment présenté sous forme agglomérée (AItKen et WiLson, I962; Braeunlich et ChemiLLIER, I966; LEBAS, I969). A notre connaissance tous les essais d'alimentation du Lapin avec un régime présenté sous forme de farine se sont soldés par des refus de consommation. Ceci oblige donc à agglomérer les aliments destinés aux lapins, ce qui n'est pas sans inconvénients, en particulier lorsqu'on prévoit l'incorporation de substances craignant l'échauffement dû à la granulation. A l'occasion de la fabrication de certains régimes expérimentaux, nous avons obtenu des rations qui nous ont semblé susceptibles d'être consommées sous forme de farine. La comparaison des performances des animaux, obtenues avec 2 types d'aliments offerts, soit en farine, soit en aggloméré, fait l'objet de la présente note. 


\section{MATÉRIEL, E'T MÉTHODE}

L'expérience a porté sur 32 lapins californiens des 2 sexes, âgés de 7 semaines au début de l'expérimentation. Cette dernière s'est poursuivie durant 4 semaines. Les animaux étaient logés individuellement dans des cages entièrement métalliques, avec une alimentation en eau automatique, par abreuvoir à clapet.

Les animaux répartis en 8 blocs de 4 ont reçu à volonté, l'un des 2 régimes définis au tableau I, soit sous forme de farine, soit sous forme d'aggloméré. Cette dernière présentation correspond simplement au passage de la farine dans une presse à granulé avec une filière de $2,5 \mathrm{~mm}$ de diamètre.

TABLEAU I

Composition centésimale des régimes

\begin{tabular}{|c|c|c|}
\hline Composants & Régime A & Régime $B$ \\
\hline Tourteau de soja $\mathbf{t}^{\prime} t$ & 一 & 25,0 \\
\hline Tourteau de soja $50 \ldots \ldots$ & 29,0 & 一 \\
\hline Maïs . . . . . . . . . & - & 58,8 \\
\hline Amidon de mais.......... & 43,0 & 一 \\
\hline Huile de mais........... & 3,0 & 一 \\
\hline Paille d'orge broyée ...... & 20,0 & 15,0 \\
\hline Glutamate de $\mathrm{Na} . . . \ldots$ & 0,8 & - \\
\hline Méthionine $\ldots \ldots \ldots \ldots$ & 0,2 & 0,2 \\
\hline Minéraux + vitamines .... & 4,0 & 4,0 \\
\hline
\end{tabular}

\section{RÉSULTATS ETT DISCUSSION}

Les performances de consommation, consignées au tableau 2, permettent tout d'abord, de constater que les animaux ont effectivement consommé les aliments présentés sous forme de farine. Compte tenu de la grande variabilité obtenue pour l'aliment $A$, du type semi-purifié, les différences ne sont pas significatives. Par contre, avec l'aliment B, de composition plus classique, la présentation d'agglomérés a favorisé significativement la consommation (tabl. 2). Pour l'aliment $B$ toujours, la granulation a tendance à légèrement réduire la variabilité de la réponse des animaux. La différence observée entre le régime $\mathrm{A}$ et le régime $\mathrm{B}$ ne constitue, à nos yeux, qu'un aspect accessoire de l'expérimentation. L'emploi de 2 régimes n'avait pour nous, que l'intérêt de vérifier que nous n'étions pas dans un cas particulier.

Ainsi, sur un régime permettant de bonnes performances (régime B) la granulation semble avoir un effet favorable. Toutefois, la conclusion essentielle de cet essai limité, reste pour nous, la possibilité de nourrir les lapins avec un aliment présenté sous forme de farine. Ainsi il s'avère possible de fabriquer, sans délai, au laboratoire, pour le lapin, comme pour le rat ou le porc, un aliment dont la composition peut varier au gré de l'expérimentateur, sans avoir recours à la granulation toujours longue à mettre en cuvre. En outre, l'utilisation de farine permet d'envisager l'emploi de rations difficiles à agglomérer ou, dont les composants risquent de s'altérer lors de la granulation. 
TABLEAU 2

Performances de croissance des lapins

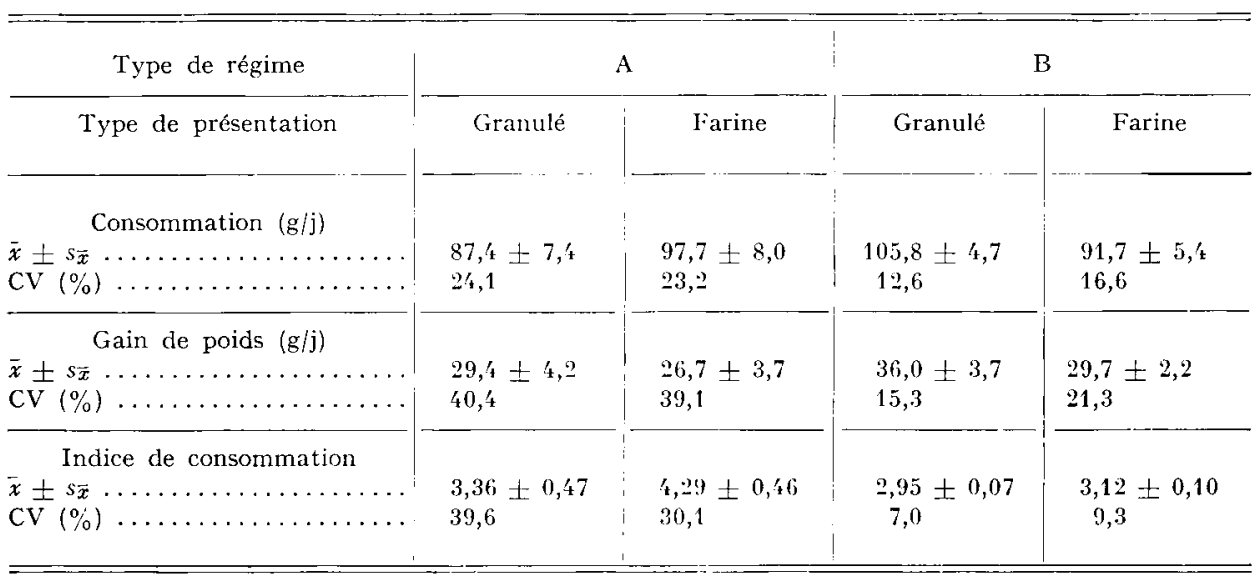

Pour expliquer les échecs observés jusqu'à présent dans les essais d'alimentation du lapin avec de la farine, on peut émettre l'hypothèse que l'emploi de luzerne ou de foins de graminées broyés, entraîne la formation de trop grandes quantités de très fines poussières nocives pour les voies respiratoires du lapin. Dans le cas des 2 régimes A et $\mathrm{B}$ que nous avons présentés, la farine n'a en effet, pas d'aspect poussiéreux.

Ręu pour publication en février 1973.

\section{SUMMARY \\ FEEDING OF GROWING RABBITS WITH MEALS \\ AS COMPARED TO PELIETS}

Two diets composed of either soybean oil-meal, maize starch, maize oil and barley straw, or of soybean oil-meal, maize and straw were offered in the form of pellets or meals to 32 young 7 weeks old rabbits. The intake of the two feeds was good during the 4 weeks of experiment independently of the physical form chosen. Pelleting seemed to have a favourable effect on growth performances. Successful utilization of meals in the feeding of rabbits could be the absence of lucerne and grass hay in the diets.

\section{RÉFÉRENCES BIBLIOGRAPHIQUES}

Aitken F. C., Wilson W. K., 1962. Rabbit feeding for meat and fur. C. A. B. Ed., Londres. 66 p. Braeunlich K., Chemillier J., I966. L'alimentation du lapin; deuxième partie : L'étude des rations Hoffman-La Roche et Cie éd., Paris. 88 p.

LEBAS F., I969. L'alimentation du lapin. L'alimentation et la vie, 57, 245-268. 